\title{
Impedance pH Guided Approach to Proton Pump Inhibitor-refractory Non-erosive Reflux Disease
}

\author{
Yu Kyung Cho
}

Department of Internal Medicine, The Catholic University of Korea, Seoul, Korea

Article: Diagnostic utility of impedance-pH monitoring in refractory non-erosive reflux disease Khan MQ, Alaraj A, Alsohaibani F, Al-Kahtani K, Jbarah S, Al-Ashgar H

(J Neurogastroenterol Motil 2014;20:497-505)

Patients with persistent symptoms despite proton pump inhibitor (PPI) make a therapeutic challenge for the clinical gastroenterologist. There are multiple mechanisms causing PPI failure; proper PPI dosing, patients compliance, functional heartburn (FH) and esophageal hypersensitivity, weakly acidic or alkaline reflux, residual acid reflux, bile acid reflux, nocturnal acid breakthrough and so on. ${ }^{1}$ Most patients with gastroesophageal reflux disease who do not respond to a PPI have either non-erosive reflux disease (NERD) or $\mathrm{FH}^{2}{ }^{2}$

Combined multichannel intraluminal impedance (MII)-pH helps clarify the association of symptoms with reflux events in patients who do not respond to PPIs. It can determine if there is ongoing pathological acid or non-acid reflux despite the PPI., Combined MII-pH has demonstrated that about one-half of patients with persistent symptoms on therapy do not have a temporal correlation between their symptoms and any type of reflux. In addition, about $40 \%$ of patients with persistent symptoms on PPI therapy have a temporal association between their symptoms and reflux, primarily of the non-acid type. ${ }^{2}$

Using impedance $\mathrm{pH}$ metry, Savarino et $\mathrm{al}^{5}$ classified NERD patients into 3 groups; $\mathrm{pH}$ positive NERD, hypersensitive esophagus and functional heartburn. ${ }^{5}$ Those with symptomatic non-acid reflux were classified as having a hypersensitive esophagus. This means that patients who earlier classified as $\mathrm{FH}$ with conventional $\mathrm{pH}$ testing were now reclassified as hypersensitive esophagus. In one study, the number of patients in the FH subgroup decreased from $43 \%$ to $26 \%$. $^{5}$ The results of impedance $\mathrm{pH}$ testing can thus guide to the next therapeutic step in management of reflux.

For patients with symptomatic non-acid reflux, either pathological NERD or hypersensitive esophagus, the therapeutic target is to decrease reflux episodes or esophageal hypersensitivity. Baclofen which reduces the transient lower esophageal sphincter relaxation, or a pain such as antidepressant can be tried. Anti-re-

Received: September 14, 2014 Revised: September 26, 2014 Accepted: September 30, 2014

(c) This is an Open Access article distributed under the terms of the Creative Commons Attribution Non-Commercial License (http://creativecommons. org/licenses/by-nc/3.0) which permits unrestricted non-commercial use, distribution, and reproduction in any medium, provided the original work is properly cited.

*Correspondence: Yu Kyung Cho, MD Department of Internal Medicine, The Catholic University of Korea, Seoul St. Mary's Hospital, 222, Banpo-daero, Seocho-gu, Seoul, Korea

Financial support: None.

Tel: +82-2-2258-6024, Fax: +82-2-2258-2055, E-mail: ykcho@catholic.ac.kr

Conflicts of interest: None. 
flux surgery can be considered as another treatment option if medical therapy is not effective and positive symptom correlation is clearly defined based on the impedance $\mathrm{pH}$ results. Based on the results of MII-pH monitoring, one study found that most patients with documented positive symptom association who underwent anti-reflux surgery, reported no symptoms during a median follow-up time of 14 months after surgery. These results indicate that MII-pH monitoring on therapy helps identify patients with persistent reflux symptoms who may benefit from anti-reflux surgery. ${ }^{6}$

The treatment options for the FH may be different. FH represents a heterogeneous group of patients with different pathophysiological mechanisms. Although a subset of patients with esophageal hypersensitivity may respond to acid suppressive therapy, this disorder is similar to the visceral hyperalgesia described in a variety of other functional gastrointestinal disorders including functional dyspepsia and irritable bowel syndrome. ${ }^{7}$

The current issue of the journal has published a prospective study $^{8}$ using MII-pH testing. Sixty symptomatic NERD patients refractory to PPI were evaluated. Approximately $60 \%$ of these patients had positive reflux-symptom association, primarily due to nonacid reflux. Nearly half of NERD patients on PPI were further sub-divided into $\mathrm{FH}$ and hypersensitive esophagus equally. The strength of this study was its prospective design. As expected, the results are not significantly different from previous studies. As mentioned in their study, cut-off reference values in classifying impedance results are still controversial. It can challenge the clinical implication of sub-classification of NERD.

In summary, combined MII-pH can help guide stepwise diagnostic approach to the patients with gastroesophageal reflux disease refractory to PPIs. However, whether the improved understanding of symptoms to reflux and non-reflux events translates into better treatment outcomes is still controversial. The approach to the pathophysiology of heterogeneous subgroups of NERD will help in making better therapeutic strategy.

\section{References}

1. Fass R, Gasiorowska A. Refractory GERD: what is it? Curr Gastroenterol Rep 2008;10:252-257.

2. Mainie I, Tutuian R, Shay S, et al. Acid and non-acid reflux in patients with persistent symptoms despite acid suppressive therapy: a multicentre study using combined ambulatory impedance-pH monitoring. Gut 2006;55:1398-1402.

3. Katz PO, Gerson LB, Vela MF. Guidelines for the diagnosis and management of gastroesophageal reflux disease. Am J Gastroenterol 2013;108:308-328.

4. Pritchett JM, Aslam M, Slaughter JC, Ness RM, Garrett CG, Vaezi MF. Efficacy of esophageal impedance $/ \mathrm{pH}$ monitoring in patients with refractory gastroesophageal reflux disease, on and off therapy. Clin Gastroenterol Hepatol 2009;7:743-748.

5. Savarino E, Zentilin $P$, Tutuian $R$, et al. The role of non-acid reflux in NERD: lessons learned from impedance-pH monitoring in 150 patients off therapy. Am J Gastroenterol 2008;103:2685-2693.

6. Mainie I, Tutuian R, Agrawal A, Adams D, Castell DO. Combined multichannel intraluminal impedance-pH monitoring to select patients with persistent gastro-oesophageal reflux for laparoscopic Nissen fundoplication. Br J Surg 2006;93:1483-1487.

7. Fass R, Sifrim D. Management of heartburn not responding to proton pump inhibitors. Gut 2009;58:295-309.

8. Khan MQ, Alaraj A, Alsohaibani F, Al-Kahtani K, Jbarah S, Al-Ashgar $\mathrm{H}$. Diagnostic utility of impedance-pH monitoring in refractory non-erosive reflux disease. J Neurogastroenterol Motil 2014; 20:497-505. 